\title{
THE RANGE OF CONDUCTION VELOCITY IN NORMAL MOTOR NERVE FIBRES TO THE SMALL MUSCLES OF THE HAND AND FOOT
}

\author{
BY \\ P. K. THOMAS, T. A. SEARS, and R. W. GILLIATT \\ From the National Hospital, Queen Square, and the Middlesex Hospital, London
}

Although measurements of nerve conduction velocity in man were first made nearly a hundred years ago, the procedure was not used clinically until 1948 when Hodes, Larrabee, and German reported reduced conduction velocity in regenerating motor nerves. Later, Hodes (1949) described slow conduction in surviving nerve fibres after poliomyelitis, and more recently it has been shown that conduction may also be slow in certain localized peripheral nerve lesions (Simpson, 1956), in polyneuritis, and in peroneal muscular atrophy (Henriksen, 1956; Lambert, 1956; Gilliatt and Thomas, 1957; Gilliatt and Sears, 1958).

The use of conduction velocity measurement as a diagnostic procedure in neurology requires a knowledge of the range of values encountered in healthy individuals. Normal values for maximum conduction velocity in human peripheral nerves have already been described by Norris, Shock, and Wagman (1953) and by Henriksen (1956), but in order to obtain a control series for our own laboratory, we have estimated maximum conduction velocity in 180 normal nerves supplying the small muscles of the hands and feet. Our own results, which are in good agreement with those of previous writers, are contained in the first part of this paper.

In estimating conduction velocity, the motor nerve trunk is stimulated at two levels and the time interval between the stimulus and the onset of the muscle action potential recorded in both instances. This interval represents conduction time in the largest and most rapidly conducting motor nerve fibres stimulated, together with a further delay at the neuromuscular junction and in the muscle itself. Provided that both stimuli are supramaximal, the difference in latency of the two muscle responses is a valid estimate of conduction time in the most rapidly conducting fibres in the nerve trunk between the two points stimulated. However, conduction time in the smaller and more slowly conducting fibres of a motor nerve cannot be measured in this way as the time of onset of their muscle action potentials is obscured by the discharge of earlier units.

This point is of some clinical importance, as it is not uncommon for patients to show values for maximum conduction velocity that are slightly below the accepted normal range; in such a case the question arises as to whether this represents conduction in abnormal fibres or whether it could be due to conduction in slow normal fibres uncovered by the loss of the larger and more rapidly conducting ones.

In order to obtain some evidence on this question, we have attempted to compare the conduction times of different nerve fibres supplying a single muscle; the results of this investigation are presented in the second part of the paper.

\section{Methods}

All tests were performed in a warm room with the subject lying on a couch and covered with blankets. In a few subjects with cold hands or feet, the limbs to be tested were immersed in hot water for five or 10 minutes before the session, but no other method of controlling temperature was attempted.

In measurements of maximum conduction velocity on 77 subjects examined at the National Hospital, the stimulus was a brief condenser discharge with a time constant of $70 \mu \mathrm{sec}$.; for 73 subjects seen at the Middlesex Hospital, a "stanco" double-channel electromyograph was used, the stimulus being a rectangular pulse of 100 $\mu \mathrm{sec}$. duration. In both instances the stimulus was delivered through an isolating transformer. For the stimulating cathodes, Walter type E.E.G.pad electrodes with a diameter of approximately $1 \mathrm{~cm}$. were used. When stimulating the median or ulnar nerve, the distal stimulating cathode was placed over the nerve trunk at a point 2 to $3 \mathrm{~cm}$. above the wrist crease; the proximal stimulus was applied 3 to $5 \mathrm{~cm}$. above the elbow. The length of nerve between the two points stimulated was estimated by surface measurement and was usually between 25 and $30 \mathrm{~cm}$. The anode was a plate electrode $2.5 \times 5 \mathrm{~cm}$. placed over the flexor surface of the forearm 
or over the deltoid insertion; these anode positions were chosen for convenience and did not affect nerve conduction time.

In the legs, the distal shock was applied to the anterior or posterior tibial nerve at the level of the ankle; the proximal shock was applied to the lateral popliteal nerve at the level of the head of the fibula or to the medial popliteal nerve immediately behind the knee joint. Surface measurement between the proximal and distal electrodes usually gave a conduction distance of 30 to $40 \mathrm{~cm}$. In these experiments the anode plate was situated either over the tibia itself or over the medial surface of the thigh just above the knee.

Muscle action potentials from the small muscles of the hands and feet were recorded through co-axial needle electrodes as described by Simpson (1956). In each case the position of the needle tip in the muscle was adjusted until the earliest response to a given stimulus was found and care was taken to ensure that the initial phase of the evoked potential was similar in appearance for both proximal and distal stimuli.

With the sweep of the cathode-ray tube locked to the stimulator, supramaximal shocks were applied once per second, the muscle response being amplified and displayed on a double-beam oscilloscope. The second beam was used to provide a time scale. Photographic superimposition of 10 successive responses was used by Simpson (1956) but in our own experience this was rarely necessary. Using brief shocks and a slow repetition rate, it was unusual for subjects to find the procedure unpleasant although stimulus intensity was supramaximal for motor fibres.

For each stimulating position, the time from the beginning of the stimulus artefact to the earliest deflection caused by the muscle response was measured and recorded in milliseconds. When the "stanco" electromyograph was used, the oscillograph sweep was triggered by the stimulus and measurement was made from the beginning of the trace. Conduction time for the segment of nerve between the two stimulating cathodes was then obtained by subtraction of the shorter from the longer figure and conduction velocity calculated in metres per second (m.p.s.).

\section{Results}

Maximum Conduction Velocity.-In the hand, observations were made on motor fibres to the abductor pollicis brevis, the abductor digiti minimi, and the first dorsal interosseous muscle. In the foot, the extensor digitorum brevis and abductor hallucis were examined. As in a previous study (Gilliatt and Sears, 1958), these control observations were made not only on healthy subjects working in the laboratory but also on the unaffected nerves of patients presenting isolated peripheral nerve lesions. In all, 180 observations were made on 150 subjects, no difference being found between staff and patients. The results obtained are given in Table $I$ and in Figs. 1 and 2.

The mean values for maximum conduction
TABLE I

MAXIMUM CONDUCTION VELOCITY FOR MOTOR FIBRES TO SMALL MUSCLES OF HANDS AND FEET

\begin{tabular}{|c|c|c|c|}
\hline \multirow{2}{*}{ Muscle } & \multirow{2}{*}{$\begin{array}{l}\text { Number } \\
\text { of } \\
\text { Subjects }\end{array}$} & \multicolumn{2}{|c|}{$\begin{array}{l}\text { Conduction Velocity } \\
\text { (m.p.s.) }\end{array}$} \\
\hline & & $\begin{array}{c}\text { Mean } \\
\text { (with S.D.) }\end{array}$ & Range \\
\hline $\begin{array}{l}\text { Abductor pollicis brevis } \\
\text { Abductor digiti minimi } \\
\text { First dorsal interosseous } \\
\text { Extensor digitorum brevis } \\
\text { Abductor hallucis }\end{array}$ & $\begin{array}{l}25 \\
46 \\
49 \\
30 \\
30\end{array}$ & $\begin{array}{l}57 \cdot 2 \div 4 \cdot 2 \\
56 \cdot 2 \pm 4 \cdot 6 \\
55 \cdot 0 \pm 4 \cdot 9 \\
49 \cdot 7 \pm 7 \cdot 1 \\
43 \cdot 2 \pm 4 \cdot 9\end{array}$ & $\begin{array}{l}51 \cdot 8-67 \cdot 1 \\
49 \cdot 0-65 \cdot 6 \\
46 \cdot 2-66 \cdot 2 \\
35 \cdot 6-63 \cdot 5 \\
36 \cdot 6-55 \cdot 9\end{array}$ \\
\hline
\end{tabular}

velocity of the motor fibres to the small hand muscles are all closely similar and statistical comparison (t test) reveals no significant difference between them. The mean value for all three muscles taken together is $56.1 \ldots 4.7$ m.p.s. In the lower limb, however, maximum conduction velocity is less and, moreover, the value for the fibres to the extensor digitorum brevis ( 49.7 m.p.s.) is appreciably greater than for the abductor hallucis $(43 \cdot 2$ m.p.s.); this difference is statistically significant $(\mathrm{t} \quad 4 \cdot 10$; $P<0.001)$.
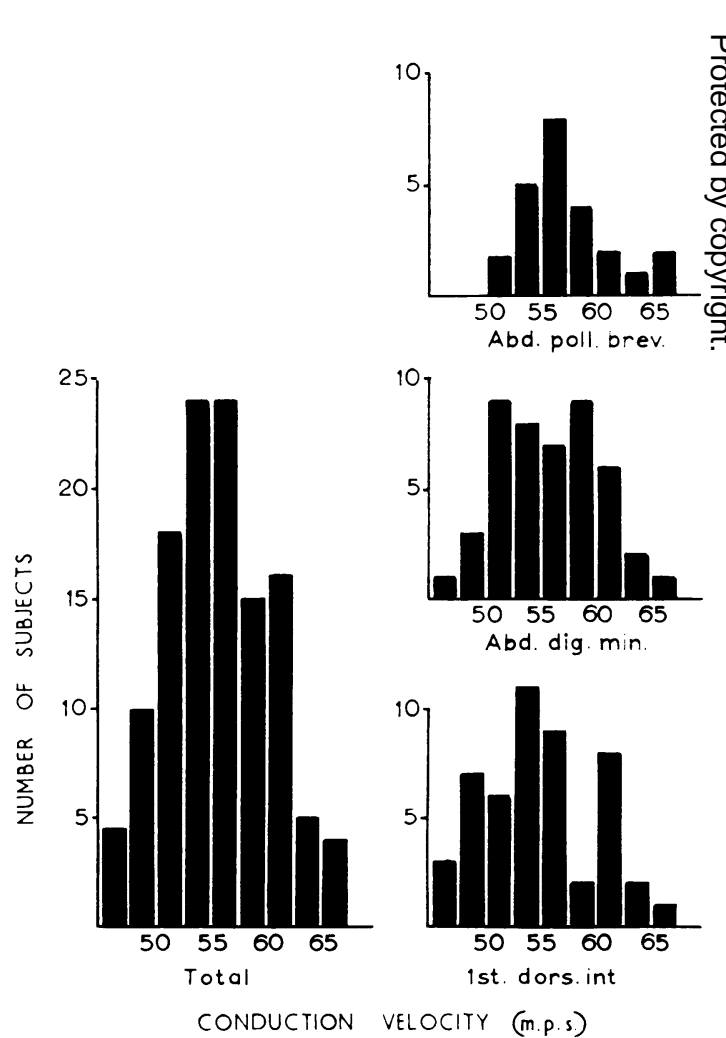

FIG. 1.-Distribution of maximum conduction velocity (in metres per second) for motor nerve fibres to the abductor digiti minimi, the first dorsal interosseous, and the abductor pollicis brevis. 


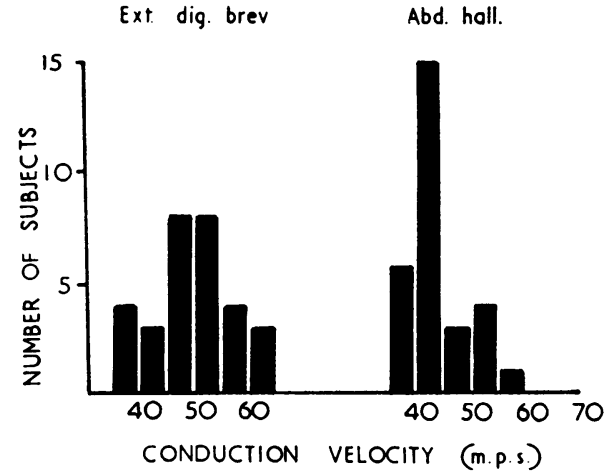

FIG. 2.-Distribution of maximum conduction velocity (in metres per second) for motor nerve fibres to the extensor digitorum brevis and the abductor hallucis.

The ages of the subjects examined are shown in Table II. Nerve conduction velocity is known to decrease in the elderly and no patients over the age of 65 were included in our series. The lower age limit was 18. From Table II it can be seen that most of the observations were on patients between the ages of 36 and 55 and that there are insufficient data from younger age groups to permit a detailed analysis of nerve conduction velocity at different ages. It is clear from Table II, however, that the differences in nerve conduction velocity between foot and hand groups cannot be explained by differences in age of the subjects tested.

TABLE II

NUMBERS OF SUBJECTS IN DIFFERENT AGE GROUPS EXAMINED

\begin{tabular}{l|c|c|c|c|c}
\hline & \multicolumn{5}{|c}{ Age Group } \\
\cline { 2 - 6 } & 18 to 25 & 26 to 35 & 36 to 45 & 46 to 55 & 56 to 65 \\
\hline $\begin{array}{l}\text { Abductor pollicis } \\
\text { brevis }\end{array}$ & 2 & 6 & 6 & 8 & 3 \\
$\begin{array}{l}\text { Abductor digiti } \\
\text { minimi }\end{array}$ & 3 & 6 & 15 & 15 & 7 \\
$\begin{array}{l}\text { First dorsal } \\
\text { interosseous }\end{array}$ & 2 & 5 & 20 & 14 & 8 \\
$\begin{array}{l}\text { Extensor digitorum } \\
\text { brevis }\end{array}$ & 0 & 6 & 10 & 13 & 1 \\
Abductor hallucis & 5 & 9 & 4 & 11 & 1 \\
\hline \multicolumn{1}{c|}{ Total } & 12 & 32 & 55 & 61 & 20 \\
\hline
\end{tabular}

Range of Conduction Velocity in Motor Fibres Supplying a Single Muscle.-Since the largest fibres in a nerve trunk have the lowest electrical threshold, a weak stimulus to the nerve is unlikely to excite small and relatively slowly conducting fibres. With a stronger stimulus the time of arrival of slowly propagated impulses at the muscle will be obscured by the discharge of earlier units. There is thus no simple method of examining the conduction rate of the slower fibres in the motor nerve trunk. (It should be pointed out that only the large motor fibres to the extrafusal muscle fibres are being considered.)

In attempting to examine the range of conduction velocity in the motor nerves to the extrafusal fibres, the most direct approach would be to compare the dispersion of the muscle action potential, recorded by surface electrodes, when the nerve is stimulated at two levels. In Fig. 4, for example, the duration of the muscle action potential of the extensor digitorum brevis is only slightly less when the motor nerve is stimulated at the ankle than when it is stimulated at the head of the fibula. This suggests that the range of conduction velocities in the motor fibres to this muscle is limited. However, this conclusion is open to objection, for Merton (1954) has shown that after electrical stimulation of a nerve trunk artificial synchronization of propagation in different muscle fibres occurs; this results in a reduction in the duration of the compound action potential.

In order to avoid this objection, it was suggested to us by Dr. Merton that if a motor nerve were stimulated proximally in the limb and the onset of the muscle response recorded in the usual way from a muscle in the hand or foot, the larger and faster motor fibres could be selectively blocked by a preceding submaximal shock to the nerve distally, thus making it possible to study the conduction time of slower fibres in isolation.

The technique is illustrated in Fig. 3, and the result of an experiment on the extensor digitorum brevis is shown in Fig. 4. The muscle action potential is recorded by surface electrodes and the test shock, $\mathrm{S} 2$, given to the lateral popliteal nerve at the knee, the stimulus being supramaximal. A preceding shock, $\mathrm{S} 1$, is applied to the nerve at the ankle a few

Fig. 3.-Arrangement of stimulating ( $\mathrm{S} 1$ and $\mathrm{S} 2)$ and recording electrodes $(R)$ for experiments to determine the range of conduction velocity in the motor nerve fibres to the extensor digitorum brevis. A maximal stimulus (S2) is preceded by a submaximal stimulus (S1) which blocks conduction in the faster and lower threshold nerve fibres. The time of arrival of the S2 volley at the muscle can be recorded for the slower nerve fibres to the muscle.

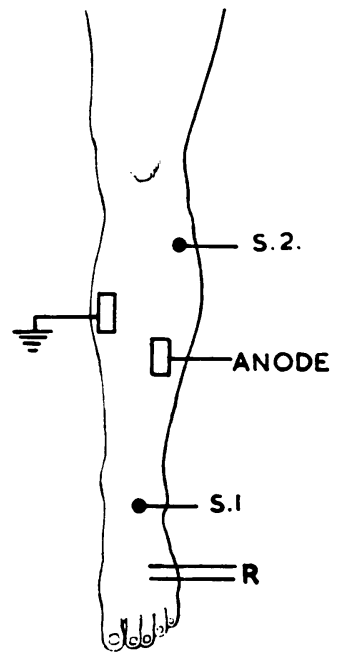



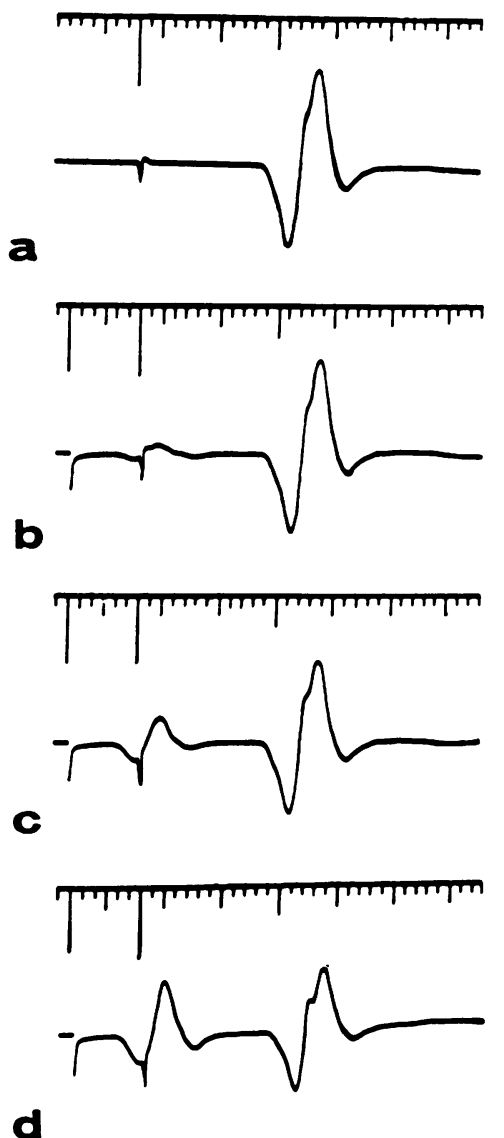
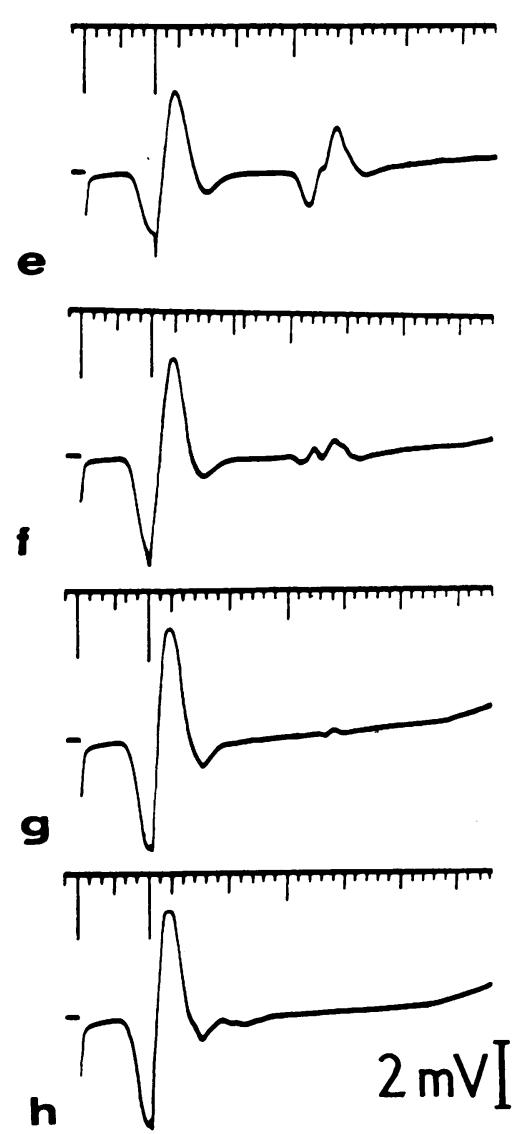

FIG. 4.-Recordings from the extensor digitorum brevis during experiments to determine the range of conduction velocity in its motor fibres. Fig. 4a shows the stimulus artefact of $\mathrm{S} 2$ followed by the muscle action potential. In Figs. $4 \mathrm{~b}$ to h, S1 precedes S2 by $6 \mathrm{~m}$.sec., the shock strength of S1 being increased progressively from threshold in Fig. $4 \mathrm{~b}$ to maximal in Fig. $4 \mathrm{~h}$. Time scale, 1 and $5 \mathrm{~m} . \mathrm{sec}$.

milli-seconds before the test shock and, if maximal, the antidromic volley will completely extinguish the descending volley from the test shock. In the experiment shown, $\mathbf{S} 1$ was arranged to precede $\mathbf{S} 2$ by $6 \mathrm{~m} . \mathrm{sec}$. in order to obtain the maximum separation between the two stimuli, conduction time between the knee and ankle being 7 to $8 \mathrm{~m} . \mathrm{sec}$. The strength of S1 was gradually increased from zero, until the muscle response to $\mathbf{S} 2$ was finally extinguished. This is illustrated in Fig. 4. In the first trace (4a), the response to $S 2$ is seen in isolation; in succeeding traces, the strength of S1 is gradually increased and the response to $\mathbf{S} 2$, is progressively eliminated, with a corresponding increase in its latency until in the final trace, the response to S1 alone remains.

Three experiments on the extensor digitorum brevis were performed in two subjects; in a further six experiments on four subjects the motor fibres supplying the abductor digiti minimi were examined in the same way, with surface recording electrodes placed over this muscle and stimulating electrodes over the ulnar nerve trunk just above the wrist and elbow. Similar results were obtained in all subjects and the results of two representative experiments are shown in Table III and in Fig. 5, in which the area of the muscle action potential (in response to $\mathrm{S} 2$ ), expressed as a percentage of its initial value, is plotted against the percentage increase in latency on increasing the strength of S1. The area was estimated, on camera lucida projections on to squared paper, by continuing the base line through the potential and measuring the area enclosed by deviations of the trace to either side of this. The curves for the foot and hand are closely similar, and it is of interest that the muscle action potential falls to about $10 \%$ of its 
initial value with only a 15 to $20 \%$ increase in latency, although in both muscles there are a small number of motor units with a latency of 30 to $40 \%$ below the maximum.

If it is accepted that the area of the compound muscle action potential is proportional to the number of motor units activated, a further point of interest is the form of the curves shown in Fig. 5. If nerve fibre size to these muscles were normally distributed, a reversed sigmoid curve might be expected, but curves of the form shown would be encountered if the distribution were markedly skewed. As an alternative explanation, it could be suggested that the larger fibres may contribute to a relatively greater extent to the area of the muscle action potential by possessing larger motor units. There is no decisive anatomical evidence on these points, although the latter possibility, at least,

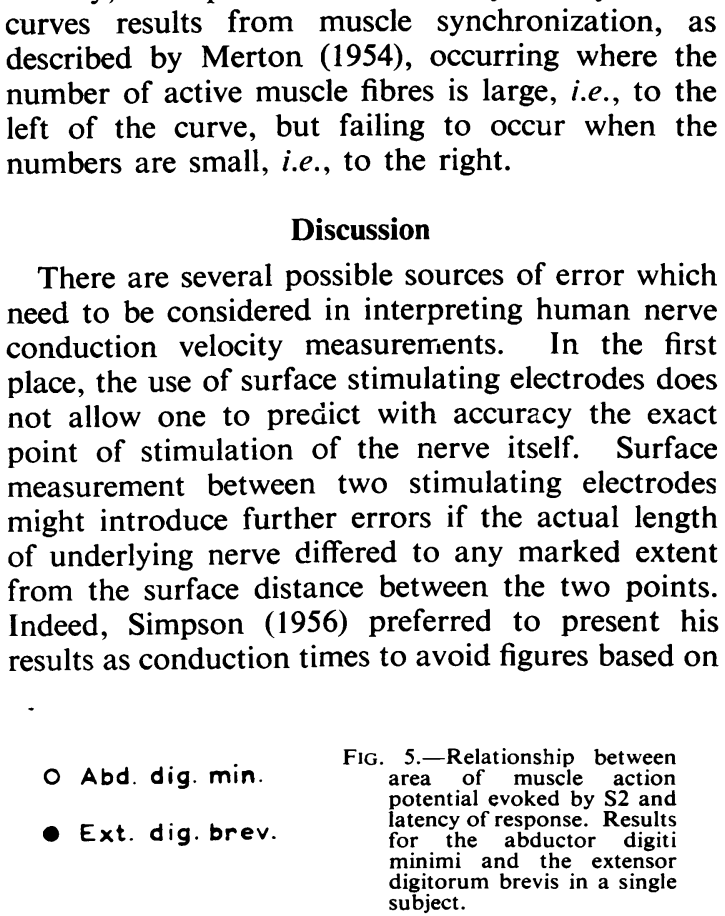
curves results from muscle synchronization, as described by Merton (1954), occurring where the number of active muscle fibres is large, i.e., to the left of the curve, but failing to occur when the numbers are small, i.e., to the right.

Discussion

There are several possible sources of error which need to be considered in interpreting human nerve conduction velocity measuremients. In the first Surface measurement between two stimulating electrodes might introduce further errors if the actual length of underlying nerve differed to any marked extent from the surface distance between the two points. Indeed, Simpson (1956) preferred to present his results as conduction times to avoid figures based on nesults for the abductor digiti minimi and the extensor digitorum brevis in a single subject.

TABLE III

RESULTS OF EXPERIMENTS SHOWN IN Fig. 4.

\begin{tabular}{|c|c|c|c|}
\hline $\begin{array}{c}\text { Stimulus } \\
\text { Intensity (S1) } \\
\text { in Volts }\end{array}$ & $\begin{array}{l}\text { Latency } \\
\text { (m.sec.) }\end{array}$ & $\begin{array}{l}\text { Percentage } \\
\text { Increase in } \\
\text { Latency }\end{array}$ & $\begin{array}{c}\text { Area of } \\
\text { Muscle Action } \\
\text { Potential } \\
\text { (\%of Initial } \\
\text { Value) }\end{array}$ \\
\hline $\begin{array}{c}\text { Abductor digiti } \\
\text { minimi } \\
0 \\
25 \\
30 \\
35 \\
37 \cdot 5 \\
40\end{array}$ & $\begin{array}{r}9 \cdot 0 \\
9 \cdot 0 \\
9 \cdot 2 \\
9 \cdot 8 \\
10 \cdot 0 \\
11 \cdot 8\end{array}$ & $\begin{array}{r}- \\
\overline{2 \cdot 2} \\
8 \cdot 9 \\
11 \cdot 1 \\
31 \cdot 1\end{array}$ & $\begin{array}{r}100 \\
97 \\
86 \\
37 \\
20 \\
3\end{array}$ \\
\hline 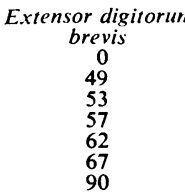 & $\begin{array}{l}10 \cdot 8 \\
10 \cdot 9 \\
11 \cdot 0 \\
11 \cdot 0 \\
12 \cdot 1 \\
12 \cdot 5 \\
15 \cdot 0\end{array}$ & $\begin{array}{r}\overline{0.9} \\
1 \cdot 9 \\
1 \cdot 9 \\
12 \cdot 0 \\
15 \cdot 7 \\
39 \cdot 1\end{array}$ & $\begin{array}{r}100 \\
97 \\
88 \\
66 \\
36 \\
13 \\
1\end{array}$ \\
\hline
\end{tabular}

seems unlikely (see Fernand and Young, 1951). Thirdly, it is possible that the asymmetry of the toes 
surface measurement. These objections are discussed in detail by both Carpendale (1956) and Henriksen (1956), who compared motor nerve conduction times during percutaneous stimulation using cathodes of different sizes and in different positions over the nerve trunk. These authors concluded that small lateral movements of the stimulating electrode did not produce significant changes in the latency of the muscle response and that with cathode diameters of up to $2 \mathrm{~cm}$. stimulation appeared to occur under the centre of the electrode. In addition, Carpendale compared the actual length of median and ulnar nerves found on dissection of the forearm with the results of surface measurements in four cadavers and concluded that the errors introduced in this way were of little importance.

A more serious difficulty is that due to differences in temperature. In our series, it is likely that temperature variation accounted for much of the observed variation between different individuals, and further experiments on this point are clearly required. In the present series, however, we have deliberately accepted variations due to uncontrolled temperature in order that the results might be directly applicable to patients seen in routine elestrodiagnostic clinics where elaborate temperature control is impracticable.

The only published investigation of the effect of temperature on conduction velocity in man is that of Henriksen (1956) who measured conduction velocity in the motor fibres of the ulnar nerve in the forearm with the arm immersed in a water bath. Temperature was recorded by a thermistor buried to a depth of $2 \mathrm{~cm}$. in the proximal third of the forearm near the course of the nerve and varied between $29^{\circ}$ and $38^{\circ}$. The mean alteration in conduction velocity was 2.4 m.p.s. per degree change in temperature. Henriksen also measured temperature during recordings made under similar conditions to those employed in our series and found that muscle temperatures for different subjects generally varied between $34^{\circ}$ and $36.5^{\circ} \mathrm{C}$.

The use of co-axial needles instead of surface recording electrodes is open to the theoretical objection that the needle electrode samples only a proportion of the motor units in a muscle, a proportion which might not be representative of the muscle as a whole. Among previous writers, Hodes, Wagman, Carpendale and Henriksen used surface electrodes, whereas Simpson preferred co-axial needles. Our own experience does not suggest that significant errors are introduced by the use of needle electrodes, at any rate in the small muscles that we have examined, and needle electrodes have clear advantages in the examination of patients with nerve lesions in that they make it possible to measure nerve conduction velocity simply and rapidly after sampling the spontaneous and voluntary activity of a paretic muscle. Furthermore, the restricted area from which the co-axial needle records is a great advantage when paretic and unaffected muscles are in close proximity. For example, during stimulation of the ulnar nerve in patients with lesions of the deep branch in the hand, the delayed response of a few surviving units in the interossei may be completely obscured in surface records by the response of unaffected hypothenar muscles. With a co-axial needle, even a single surviving unit in an affected muscle can be localized by exploration during voluntary contraction and then observed in isolation during nerve stimulation.

While our results show no significant difference in maximum conduction velocity of motor fibres to the abductor digiti minimi, the abductor pollicis brevis, and the first dorsal interosseous of the hand, the differences in velocity we have observed between the hand and foot and between fibres supplying the extensor digitorum brevis and the abductor hallucis call for comment. It is possible that the lower values obtained in the leg as compared with the arm may be accounted for in terms of a temperature effect, although this has not been tested. However, a temperature effect is unlikely to explain the disparity between the two muscles in the foot. The segments of the anterior and posterior tibial nerves over which the recordings were made are both deeply buried in muscle and a difference between the two nerves is thus improbable. In searching for an alternative explanation it will be recalled that the diameter of the anterior root fibres is greatest at the centre of the lumbar enlargement, diminishing progressively on either side of this but particularly caudally (Häggqvist, 1937; Gentele and Swensson, 1941). Since the abductor hallucis receives its innervation from more caudal segments (S1, S2) than the extensor digitorum brevis (L4, L5, S1), the fibres supplying it are thus likely to be of smaller diameter.

The scatter of conduction velocities to the abductor digiti minimi and the extensor digitorum brevis in a single subject indicates that the majority of fibres conduct at a rate within 15 to $20 \%$ of the maximum, although the lower limit of velocity may be as much as 35 to $40 \%$ below the maximum. In the rabbit, the distal muscles in the limbs are innervated by fibres of small diameter and with a restricted size range (Fernand and Young, 1951), and it seems likely that this observation is generally applicable. If this is also the situation in man, it would account for the relatively restricted range of conduction velocity in the motor nerve fibres to these muscles. 
It is of interest to relate these findings to those obtained in patients with lower motor neurone lesions. Conduction velocity in surviving fibres after anterior poliomyelitis or in motor neurone disease may be reduced (Hodes, 1949; Henriksen, 1956) but values more than $30 \%$ below normal have not been recorded. This degree of slowing could well be accounted for in terms of conduction in surviving slow normal fibres. On the other hand, some patients with polyneuritis and peroneal muscular atrophy may show extremely low values for nerve conduction velocity, at times below 10 metres per second. This clearly cannot be explained in terms of slow normal surviving fibres and must mean abnormal nerve function. Whether this depends upon structural or upon metabolic alterations, or upon both, has not yet been ascertained.

\section{Summary}

Conduction velocity in the fastest motor nerve fibres to the small muscles of the hand and foot was calculated for a series of normal nerves, measurements being made for fibres to the abductor pollicis brevis, the abductor digiti minimi, and the first dorsal interosseous muscle in the hand, and the extensor digitorum brevis and abductor hallucis in the foot. There was no significant difference between the hand muscles, the mean value obtained being $56.1 \pm 4.7 \mathrm{~m}$.p.s. The values obtained for the foot were less, being $49.7 \pm 7 \cdot 1$ m.p.s. for the extensor digitorum brevis and $43 \cdot 2 \pm 4 \cdot 9$ m.p.s. for the abductor hallucis, the difference between these two muscles being statistically significant.

A method is described for measuring conduction velocity for the slower motor fibres to a muscle, and observations on the abductor digiti minimi and the extensor digitorum brevis indicate that there are motor fibres supplying these muscles with a conduction velocity between $30 \%$ and $40 \%$ below the maximum. The significance of this finding is discussed.

We wish to thank Dr. W. A. Cobb and Dr. G. D. Dawson for helpful criticism and advice, and Mr. H. B. Morton for technical assistance.

\section{REFERENCES}

Carpendale, M. T. F. (1956). M.S. (Phys. Med.) Thesis, Univ. of Minnesota.

Fernand, V. S. V., and Young, J. Z. (1951). Proc. roy. Soc. B., 139. 38.

Gentele. H., and Swensson, A. (1941). Z. mikr.-anat. Forsch., 50, 190.

Gilliatt, R. W., and Sears, T. A. (1958). J. Neurol. Neurosurg. Psychiat., 21, 109

- and Thomas, P. K. (1957). Ann. phys. Med., 4, 104.

Häggqvist, G. (1937). Z. mikr.-anat. Forsch., 42, 33.

Henriksen, J. D. (1956). M.S. (Phys. Med.) Thesis. Univ. of Minnesota.

Hodes, R. (1949). J. Neurophvsiol, 12, 257.

-, Larrabee, M. G., and German, W. (1948). Arch. Neurol. Psychiat, (Chicago), 60 340.

Lambert, E. H. (1956). In Clinical Examinations in Neurology, p. 313. Saunders, Philadelphia.

Merton, P. A. (1954). J. Physiol. (Lond.), 124, 311.

Norris, A. H., Shock, N. W., and Wagman, I. H. (1953). J. appl Physiol., 5, 589.

Simpson, J. A. (1956). J. Neurol. Neurosurg. Psychiat., 19, 275. 